\title{
A phage-based decolonisation strategy against pan-resistant enterobacterial strains
}

An outbreak of carbapenemase-producing and colistinresistant Klebsiella pneumoniae was reported in north-east Germany in 2019. ${ }^{1}$ The alert raised by the European Centre for Disease Prevention and Control on Oct 28, 2019, ${ }^{1}$ emphasised that a pandrug-resistant $K$ pneumoniae clone had spread in three hospitals and one rehabilitation clinic, with specimens recovered from 17 patients (six infected and 11 colonised). ${ }^{1}$ The strain showed a broad resistance pattern to most antibiotics; it was resistant to all betalactams (including ceftazidime-avibactam) because of co-production of two carbapenemases, NDM-1 and OXA-48. Furthermore, the strain was resistant to fluoroquinolones, aminoglycosides, and polymyxins. The only available therapeutic options (according to in-vitro susceptibility testing) were fosfomycin and tigecycline. Moreover, the outbreak strain was shown to belong to sequence type ST307, which is recognised as an internationally emerging high-risk clone in health-care-associated outbreaks, possessing a series of well-recognised virulence factors. ${ }^{2}$ Effective control measures were taken and dissemination of the strain was, presumably, controlled since no additional cases have been reported in Germany since the end of September, 2019.

Efficient strategies to prevent further dissemination of a multidrug-resistant enterobacterial strain include reinforced hygiene protocols, grouping carriers into a cohort, dedicated health-care workers, systematic rectal screening of contact patients, and pre-emptive isolation of individuals until screening results are available. Decolonisation strategies can also be considered in some situations. Selective digestive decontamination strategies, particularly those using colistin, might be efficient to partly decolonise patients. However, once interrupted, they do not prevent re-establishment of the targeted strain in the gut reservoir. Furthermore, a decolonisation strategy has an important pitfall because it might select for colistin-resistant strains, which is a very concerning collateral effect.

Besides selective digestive decontamination, a new approach has been proposed. A bacteriophage therapy was used successfully in Italy for eradication of a multidrug-resistant and carbapenemase-producing $K$ pneumoniae strain from the gut of a patient who was colonised for a long period ( 6 months) and who had several breakthrough episodes of clinically invasive infections sustained by this microorganism. The approach used was an oral plus intra-rectal therapy using a customised lytic bacteriophage preparation. ${ }^{3}$ The $K$ pneumoniae strain belonged to ST307, similar to the NDM- 1 and OXA-48 outbreak strain, but differed by the type of carbapenemase produced (KPC-3). ${ }^{3}$

In view of the successful eradication of the KPC-3-producing and ST307 $\mathrm{K}$ pneumoniae strain in Italy, we were prompted to assess the efficacy of the bacteriophage, called vB_KpnM_GF, which belongs to the Myoviridae family, ${ }^{3}$ against a representative isolate (namely, NRZ-52340) of the outbreak strain from Germany. The lytic activity of the vB_KpnM_GF phage was tested using the soft-agar overlay method. ${ }^{4}$ An equivalent growth inhibition zone was seen with strain NRZ-52340 compared to the KPC-3-producing isolate, indicating that strain NRZ-52340 was susceptible to the lytic action of this phage. Thus, invivo significant activity could possibly be achieved in a similar manner to that noted with the Italian case, in the context of a decolonisation strategy. Therefore, use of bacteriophages might contribute an option for management of the strain from Germany. Indeed, such a strategy might have two main benefits if the NDM- 1 and OXA-48 K pneumoniae strain was present in different patients. First, a decolonisation strategy using bacteriophages might constitute an individual benefit for persistent carriers of the microorganism, who might develop systemic infections caused by this difficult-totreat isolate. Second, successful decolonisation might constitute a general benefit by reducing the number of colonised patients, therefore limiting further spread of the pan-resistant microorganism in health-care settings.

We declare no competing interests. This work was partly funded by a grant from the Laboratoire Européen Associé, Institut National de la Santé et de la Recherche Médicale (INSERM) and the University of Fribourg (Fribourg, Switzerland). We also acknowledge financial support provided by the Eliava Foundation (Tbilisi, Georgia) and by the Associazione Nazionale per la Lotta all' AIDS (ANLAIDS, Sezione Lombarda, Milan, Italy).
Published Online March 5, 2020 https://doi.org/10.1016/ S1473-3099(20)30140-7 
*Laurent Poirel, Patrice Nordmann, José Manuel Ortiz de la Rosa, Mzia Kutateladze, Sören Gatermann, Mario Corbellino laurent.poirel@unifr.ch

Medical and Molecular Microbiology Unit, Department of Medicine, Faculty of Science, University of Fribourg, CH-1700 Fribourg, Switzerland (LP, PN, JMOR); INSERM European Unit (IAME, France), University of Fribourg, Fribourg, Switzerland (LP, PN); Swiss National Reference Center for Emerging Antibiotic Resistance (NARA), University of Fribourg, Fribourg, Switzerland (LP, PN); Institute for Microbiology, University of Lausanne and University Hospital Centre, Lausanne, Switzerland (PN); G Eliava Institute of Bacteriophages, Microbiology and Virology, Tbilisi, Georgia (MK); Ruhr-Universität Bochum, Department of Medical Microbiology, Bochum, Germany (SG); and III Division of Infectious Diseases, ASST Fatebenefratelli - Sacco, Milan, Italy (MC)
1 European Centre for Disease Prevention and Control. Outbreak of carbapenemase-producing (NDM-1 and OXA-48) and colistin-resistant Klebsiella pneumoniae ST307, north-east Germany, 2019. Oct 28, 2019. https://www.ecdc.europa.eu/en/publications-data/outbreak-Klebsiellapneumoniae-Germany (accessed Feb 17, 2020).

2 Villa L, Feudi C, Fortini D, et al. Diversity, virulence, and antimicrobial resistance of the KPC-producing Klebsiella pneumoniae ST307 clone. Microb Genom 2017; 3: e000110.

3 Corbellino M, Kieffer N, Kutateladze M, et al. Eradication of a multi-drug resistant, carbapenemase-producing Klebsiella pneumoniae isolate following oral and intra-rectal therapy with a custom-made, lytic bacteriophage preparation. Clin Infect Dis 2019; published online Aug 15. DOI:10.1093/cid/ciz782.

4 Adams MH. Bacteriophage. New York: Interscience Publishers, 1959

\section{(1) Transdisciplinary work against antimicrobial resistance}

Published Online

March 5, 2020

https://doi.org/10.1016/ S1473-3099(20)30137-7
In 2015, WHO issued a Global Action Plan emphasising One Health as an essential approach to tackle antimicrobial resistance (AMR). ${ }^{1}$ One Health aims to bridge human, animal, and environmental sectors to address shared health concerns. ${ }^{2}$ One of the gaps in knowledge articulated by the Global Action Plan is scant understanding of social science and behaviour. In that context, One Health offers a potential for transdisciplinary collaborations, including between social and biomedical disciplines. WHO states that the role of social sciences in the One Health approach to AMR is dedicated to support effective antimicrobial stewardship programmes in human and animal health and agriculture. Although this role is essential for the success of AMR control programmes, it limits the potential of social science studies to support such programmes rather than to co-develop them based on both biomedical and social knowledge.

Also in 2015, Smith ${ }^{4}$ wrote that AMR is a social problem; social science research has to be taken seriously in addressing the issue of AMR. Cultures of prescription, sale, and use of antibiotics in human and animal sectors, and practices of antibiotic production and waste management, are essential for understanding drivers of emergence and dissemination of multidrugresistant bacteria..$^{5-7}$ Study findings substantiate the claim that AMR is not only a biological problem but also a social problem. Research by Collignon and colleagues ${ }^{8}$ shows that social and economic inequalities, poverty, and public health expenditures are major factors driving the global level of AMR.

If AMR can be considered a biosocial issue, solutions should neither lie exclusively within the biomedical disciplines nor fall into the social disciplines, but should reside on their intersections. In principle, the One Health approach offers an opportunity to develop a transdisciplinary and trans-sectoral agenda for AMR. However, findings of a study ${ }^{3}$ suggest that the One Health governing framework has an anthropocentric focus, positioning animal and agricultural sectors under the dominance of human health governing organisations. Moreover, at the moment there are not enough conceptual, physical, and financial infrastructures to undertake transdisciplinary and transsectoral work. To create possibilities for transdisciplinary research, combining sociological and anthropological studies with microbiological research, funding needs to be organised; moreover, opportunities are needed to publish the results of transdisciplinary research, which combines very different types of data. It is important to think about concepts and approaches that go beyond One Health in capturing the multiple biosocial complexity of AMR, without prioritising one discipline over the other. Without a research infrastructure to build and sustain transdisciplinary collaborations, we are locked in disciplinary paradigms and will not understand AMR as a biosocial issue.

We declare no competing interests.

${ }^{*}$ Alena Kamenshchikova, Petra F G Wolffs, Christian J P A Hoebe, Klasien Horstman a.kamenshchikova@maastrichtuniversity.n

Department of Health, Ethics and Society (AK, KH), and Department of Social Medicine and Medical Microbiology (CJPAH), School of Public Health and Primary Care (CAPHRI), Maastricht University, Maastricht 6211 LK, Netherlands; Department of Medical Microbiology, CAPHRI, Maastricht University Medical Center (MUMC+), Maastricht, Netherlands (PFGW); and Department of Sexual 